\title{
3-D relative positioning sensor for indoor flying robots
}

\author{
James F. Roberts • Timothy Stirling • \\ Jean-Christophe Zufferey • Dario Floreano
}

Received: 31 May 2011 / Accepted: 21 January 2012 / Published online: 8 February 2012

(C) Springer Science+Business Media, LLC 2012

\begin{abstract}
Swarms of indoor flying robots are promising for many applications, including searching tasks in collapsing buildings, or mobile surveillance and monitoring tasks in complex man-made structures. For tasks that employ several flying robots, spatial-coordination between robots is essential for achieving collective operation. However, there is a lack of on-board sensors capable of sensing the highlydynamic 3-D trajectories required for spatial-coordination of small indoor flying robots. Existing sensing methods typically utilise complex SLAM based approaches, or absolute positioning obtained from off-board tracking sensors, which is not practical for real-world operation. This paper presents an adaptable, embedded infrared based 3-D relative positioning sensor that also operates as a proximity sensor, which is designed to enable inter-robot spatial-coordination and goal-directed flight. This practical approach is robust to varying indoor environmental illumination conditions and is computationally simple.
\end{abstract}

Keywords Relative positioning sensing - Indoor flying robots $\cdot$ Collective operation $\cdot 3 \mathrm{D}$ sensor .

Spatial-coordination $\cdot$ Proximity sensing

J.F.R. developed the concept of relative positioning sensing for enabling goal-directed flight on indoor collective flying robots, wrote the manuscript, developed the sensor hardware/firmware, developed the calibration tools and characterised the sensor.

T.S. extensively contributed to the sensor firmware and characterisation.

J.-C.Z. and D.F. conceived and directed the project sponsoring the work described in the article. They also provided continue support and feedback towards reaching the attained results.

J.F. Roberts $(\bowtie) \cdot$ T. Stirling · J.-C. Zufferey · D. Floreano

Ecole Polytechnique Fédérale de Lausanne (EPFL), Laboratory of Intelligent Systems (LIS), Station 11, Lausanne 1015, Switzerland

e-mail: contact@jfroberts.com

\section{Introduction}

Indoor flying robots are promising for many real-world applications, due to their high-manoeuvrability and powerful remote sensing capability. Small flying robots could be deployed within complex man-made structures, such as a collapsing building, to search for injured people. They could also be used in warehouses or factories to monitor chemicals or radioactive materials that would be too dangerous for human contact.

Swarms of indoor flying robots allow for efficient parallel operation and are robust due to redundancy (Sahin 2005). For tasks that employ several flying robots, spatialcoordination between robots is essential for achieving collective operation. There are numerous multi-robot spatialcoordination algorithms recently developed that assume absolute or relative positioning information between robots is available. Such algorithms range from simple interrobot collision avoidance behaviours (Hoffmann and Tomlin 2008) to more complex behaviours such as flocking (Pilz et al. 2009) or chain formation (Stirling et al. 2010). GPS cannot be used indoors, as reception is poor and the positioning is unreliable (Rudol et al. 2008). In order to solve this indoor spatial-coordination problem, it is necessary to find alternative sensing methods.

Flying autonomously indoors, such as in office buildings, is very challenging, as there are many obstacles to avoid such as walls, furniture and people, thus proximity sensing is also a necessity. The width of corridors and narrow doorways creates a strong platform size limitation (standard doorway width is approximately $1.0 \mathrm{~m}$ ) that leads to a limited payload capacity (Soundararaj et al. 2009; Grzonka et al. 2009) and short flight endurance (Roberts et al. 2008; Valenti et al. 2007). 


\subsection{Related work}

Due to the difficulty in developing small, light-weight and precise on-board sensors, many researchers are using offboard tracking sensors (Valenti et al. 2007; Lupashin et al. 2010; Kirchner and Furukawa 2005) to achieve spatialcoordination and goal-directed flight. Impressive results have been achieved, however external sensing systems like these are not viable methods for real-world applications in unknown environments.

Other researchers are working with on-board vision systems and/or laser scanners that can extract motion information using feature tracking algorithms (Achtelik et al. 2009; Guenard et al. 2008; Grzonka et al. 2009). This type of sensing is far more practical, however to achieve spatialcoordination between multiple robots a method such as Simultaneous Localisation and Mapping (SLAM) (Bachrach et al. 2009; Blösch et al. 2010) would be required to extract and compare each robots position and orientation information. Such an approach is possible, however it is computationally expensive and requires a fast processor (e.g. 1.6 GHz) for real-time on-board processing (Shen et al. 2011; Weiss et al. 2011).

Alternatively, simple relative positioning sensors that can detect the position and orientation between robots, can provide all the information that is necessary for autonomous spatial-coordination.

Various relative positioning technologies are available, which have been developed for ground based robots, each with their own benefits and limitations. For example, ultrasound can be used to achieve high accuracy range $(5 \mathrm{~cm}$ max.) and bearing $\left(10^{\circ}\right.$ max. $)$ measurements using Time-ofFlight (TOF) sensing (Shoval and Borenstein 2001; Rivard et al. 2008). However, the refresh rate is relatively slow, up to $1.33 \mathrm{~Hz}$ for 10 robots, due to the slow propagation of the sound signal (Rivard et al. 2008). Alternatively, infrared (IR) can be used to achieve faster refresh rates but at the cost of a reduced accuracy. Pugh et al. (2009) have developed an eight photodiode infrared (IR) relative positioning sensor with a fast refresh rate $(25 \mathrm{~Hz}$ for 10 robots), however the maximum operating range is only $3.3 \mathrm{~m}$. The infrared (IR) relative positioning sensor by Kemppainen et al. (2006), uses a mechanically rotating single receiver to obtain bearing information, and has a variable gain receiver to achieve a long operating range $(10 \mathrm{~m})$. However, the design is not scalable due to the use of frequency division multiplexing, it also has a slow refresh rate $(0.5 \mathrm{~Hz})$ and may have reliability issues due to the rotating mechanics.

Breitenmoser et al. (2011) have developed a relative positioning system that consists of two complementary modules: a monocular vision module and a target module with four active or passive markers. The system essentially allows for the position and orientation of a small number of robots to be determined, as long as every robot has unique target markers that are dissimilar from all directions. A clear limitation of the approach is shown in cluttered environments of changing light and colour, where the markers are not easily detectable. Additionally, the system is directional (dependant on the camera viewing angle), suffers from occlusion, and has a limited range that is determined by the size of the target markers, and the resolution of the camera (approximately $3 \mathrm{~m}$ maximum range with $3 \mathrm{~cm}$ passive markers and a $752 \times 480$ resolution camera). Many other systems exist that depend on complex processing with cameras (Nakamura et al. 2003) or other heavy hardware (Montesano et al. 2004) that are unsuitable for a small flying robot.

To the best of our knowledge, the only on-board relative positioning system demonstrated on an indoor flying robot, is presented by Melhuish and Welsby (2002) for use on a swarm of lighter-than-air vehicles. In their work, a long range $(20 \mathrm{~m})$ infrared relative positioning sensor is used to achieve a simple gradient ascent behaviour towards an emitting beacon. However, due to the small payload available on an indoor lighter-than-air vehicle (limited by a $1.0 \mathrm{~m}$ doorway opening) and its very slow dynamics, their sensor was reduced to only $180^{\circ} 2-\mathrm{D}$ sensing. Apart from the system by Breitenmoser et al. (2011), all of the existing relative positioning sensors give only planar 2-D information. This 2-D information is useful for robots operating on the ground and for flying robots with slow dynamics that stay at the same height and do not tilt their body as they fly. However, for flying robots that have fast dynamics (e.g. helicopters and quad-rotors), fast and high accuracy sensing is required. During flight, these platforms can tilt as they translate and the difference in altitude can vary by several meters. Therefore, to achieve robust sensing for highly-dynamic indoor flying robots, it is necessary to have a 3-D sensor coverage.

Relative positioning could be used in most situations, as it does not rely on feature extraction in the surrounding environment like SLAM based (laser scanner and vision) approaches and can be computationally simple (when using signal strength or TOF based sensing). However, there is a lack of embedded sensors, commercially available or in research that can provide on-board 3-D relative positioning, and orientation information between multiple indoor flying robots.

The aim of the presented work was to design and test an infrared 3-D relative positioning sensor that is capable of sensing the range, bearing and elevation between indoor flying robots and their respective yaw orientations, which can also provide proximity sensing in a 3-D space. Such a sensor can potentially enable goal-directed flight and collective operation between multiple flying robots, within real-world environments.

This paper builds on a previously developed 2.5-D relative positioning sensor (Roberts et al. 2009). In order for the sensor to be suitable for highly-dynamic flying robots, 
Fig. 1 Example diagram showing the idealised 3-D geometry of one sensor receiving (left) and another sensor transmitting (right), where both are represented as spheres. As an approximation, the received signal strength $(s)$ is proportional to the inverse square of the range $(r)$. The photodiode signal with the strongest strength is defined as $s_{0}$, the angular spacing $(\beta)$ of the photodiodes is $\frac{\pi}{4}$ and the number of photodiodes about each axis is $N=8$. The bearing and elevation offset angles can be triangulated using the three photodiode signal values

$\left(s_{-1,0,1}\right)$ along the corresponding plane (where $m=1$ )

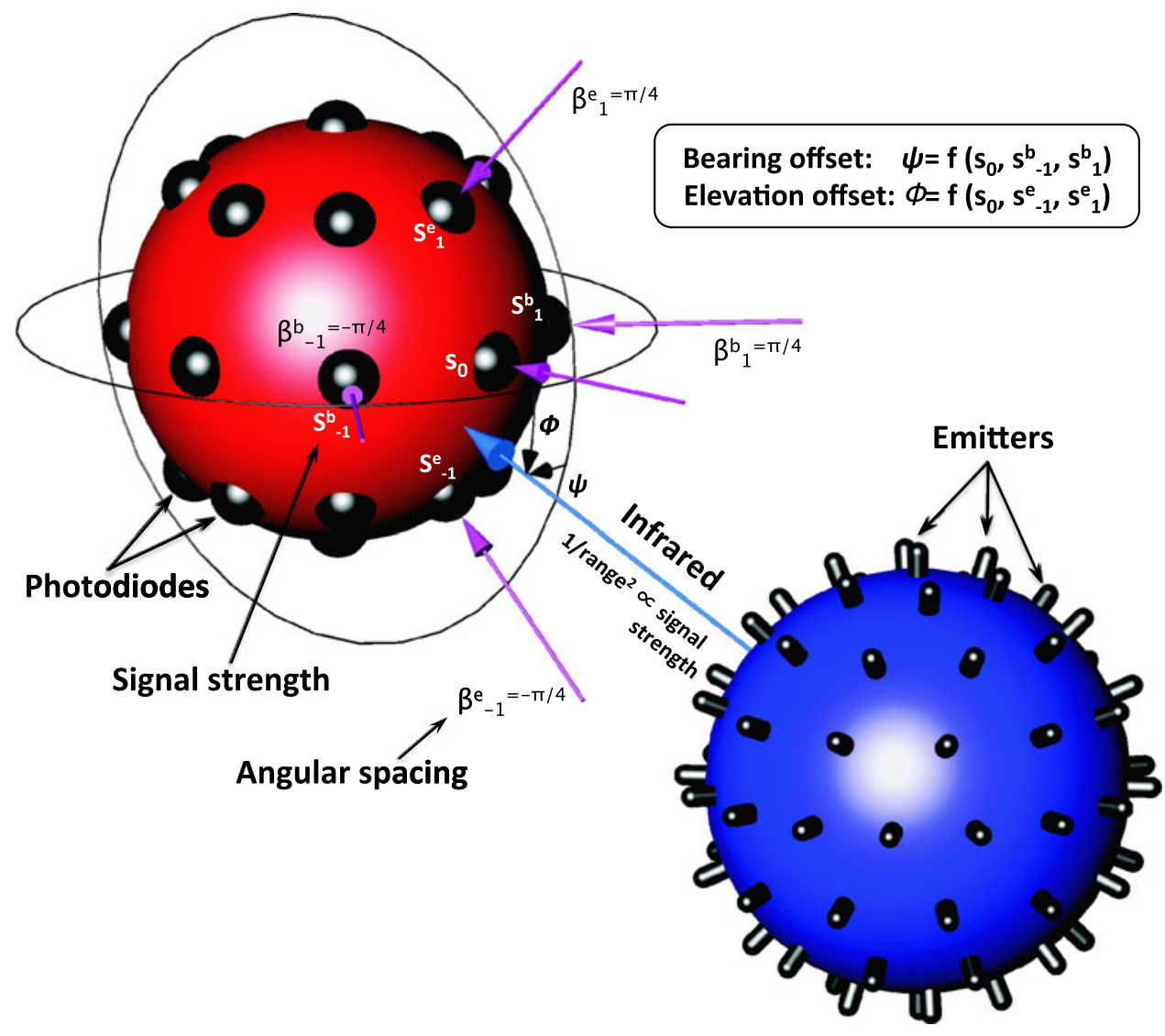

it has been extended to 3-D and the yaw orientation of the transmitting robot is now available, which is necessary for full spatial-coordination. The new sensor includes spherical proximity sensing, has more than twice the operating range and an improved error performance.

First the underlying functioning principle of the developed 3-D relative positioning sensor is introduced, to explain how it operates. The details of the sensor are then discussed, including the communication algorithm, the infrared transmission and the reception process. The physical constraints and the possible layouts of the sensor are then described, outlining suitable designs for flying robots. The 3-D relative positioning sensor is then extensively characterised to show its performance against the best three 2-D relative positioning sensors found in the literature. The light immunity and proximity sensing was also tested to observe the sensor's ability to be used for obstacle avoidance and within indoor environments with large changes in ambient lighting, respectively.

\section{Functioning principle}

To explain the functioning principle of the 3-D relative positioning sensor, two sensors can be represented as spheres, where one is transmitting and the other is receiving infrared signals. In this example the emitting and receiving functionalities are represented separately, however during normal operation each sensor will implement both functions. The receiving sensor must be able to detect the relative range, bearing and elevation of the transmitting sensor within a 3-D space. An example diagram showing the 3-D geometry can be seen in Fig. 1. Ideally, the transmitting sensor emits infrared equally in every direction. As the infrared signal propagates through the air the signal is attenuated. As an approximation, the received signal strength is proportional to the inverse square of the range between the two sensors (Naboulsi et al. 2005). However, to obtain the best performance, the signal strength from each photodiode can be linearised and converted into a range using a linear interpolator and a lookup table. This is essential when using the proposed cascaded filtering technique, as explained in Sect. 2.3. The receiving sensor detects this emitted signal on multiple photodiodes and can triangulate the bearing and elevation angles towards the transmitting sensor.

In order to calculate the 3-D relative range, bearing and elevation to the transmitting sensor, a variation of the 2-D (range and bearing) sensor algorithm as described by Pugh et al. (2009) is employed. This algorithm assumes that both the bearing and elevation angles are calculated using only planar spaced photodiodes that are angled equally about the corresponding axis. The geometry of the photodiodes and 
their angular sensitivity determines the way in which the bearing and elevation angles are calculated.

Observing the angular sensitivity of the photodiodes ${ }^{1}$ shows that it can be modelled by the cosine function using the angle of incidence to the transmitter. This is a good approximation over the whole range of the angular sensitivity, however for the lower angles (below $\frac{\pi}{8}$ ), the square-root of the cosine function is a better approximation. This relates to an improved near-field response at the cost of a slightly reduced far-field performance. The relative positioning between flying robots is more critical when flying in close proximity, thus the square-root approximation has been chosen.

Therefore, if $s$ is the signal strength of the photodiode when directly facing the transmitter, the signal strength $\left(s^{\prime}\right)$ with respect to the angle of incidence to the transmitter $(\theta)$ can be approximated by:

$s^{\prime}=s \sqrt{\cos (\theta)}$

During a transmission approximately half of the photodiodes around the sphere will detect a signal. Thus, the "area of interest" can be defined as a set of $m$ photodiodes with the highest received signals, where $m$ is no greater than half of the number of photodiodes around the sphere. Let the number of photodiodes about each axis be defined as $N=8$. The photodiode signal closest to the centre of this area, i.e. with the strongest signal strength, is defined as $s_{0}$. The horizontal planar photodiodes corresponding to the bearing offset angle $(\psi)$ have a clockwise or counter-clockwise angle from the centre of the receiving area, whereas the vertical planar photodiodes corresponding to the elevation offset angle $(\phi)$ have a higher or lower angle than the centre of the receiving area. Let the photodiode signals that have a higher or counter-clockwise angle have a positive index: $s_{1}, \ldots, s \frac{m}{2}$, and the photodiode signals that have a lower or clockwise angle have a negative index: $s_{-1}, \ldots, s_{-\frac{m}{2}}$. As a simplification the bearing and elevation angles are treated independently. The photodiode signals for the corresponding horizontal or vertical plane, can then be defined as:

$s_{i}^{b}=s_{b} \sqrt{\cos \left(\psi+\beta_{i}\right)}, \quad s_{i}^{e}=s_{e} \sqrt{\cos \left(\phi+\beta_{i}\right)}$

with $\beta_{i}=-\beta_{-i}$, and $s_{0}=s_{0}^{b}=s_{0}^{e}$

where $\beta_{i}$ is the angular offset of photodiode $i$ from the centre of the area.

By rearranging the equation to incorporate the signal values from multiple photodiodes about the bearing axis, it can be derived that:

$$
\begin{aligned}
s_{-i}^{b 2}+s_{i}^{b 2} & =s_{b}^{2} \cos \left(\psi-\beta_{i}\right)+s_{b}^{2} \cos \left(\psi+\beta_{i}\right) \\
& =2 s_{b}^{2} \cos (\psi) \cos \left(\beta_{i}\right)
\end{aligned}
$$

\footnotetext{
${ }^{1}$ http://www.vishay.com (BPV22NF, accessed Feb. 2011).
}

$$
\begin{aligned}
s_{-i}^{b 2}-s_{i}^{b 2} & =s_{b}^{2} \cos \left(\psi-\beta_{i}\right)-s_{b}^{2} \cos \left(\psi+\beta_{i}\right) \\
& =2 s_{b}^{2} \sin (\psi) \sin \left(\beta_{i}\right)
\end{aligned}
$$

Similarly, for the elevation axis:

$s_{-i}^{e 2}+s_{i}^{e 2}=2 s_{e}^{2} \cos (\phi) \cos \left(\beta_{i}\right)$

$s_{-i}^{e 2}-s_{i}^{e 2}=2 s_{e}^{2} \sin (\phi) \sin \left(\beta_{i}\right)$

In order to find the angle of the incoming signal, the bearing axis photodiode signals need to be combined (where $i=1$ to match the example):

$a=\frac{s_{1}^{b 2}+s_{-1}^{b 2}}{2 \cos (\beta)}=s_{b}^{2} \cos (\psi), \quad b=\frac{s_{1}^{b 2}-s_{-1}^{b 2}}{2 \sin (\beta)}=s_{b}^{2} \sin (\psi)$

Similarly, for the elevation axis:

$c=\frac{s_{1}^{e 2}+s_{-1}^{e 2}}{2 \cos (\beta)}=s_{e}^{2} \cos (\phi), \quad d=\frac{s_{1}^{e 2}-s_{-1}^{e 2}}{2 \sin (\beta)}=s_{e}^{2} \sin (\phi)$

This technique can be generalised for setups with finer angular discretisation by summing the additional photodiode signals from $i=1$ to $\frac{m}{2}$.

The bearing $(\psi)$ and elevation $(\phi)$ angular offsets from the centre of the segment can then be calculated using the photodiode signals from their corresponding planes:

$\psi=\arctan \frac{b}{a}, \quad \phi=\arctan \frac{d}{c}$

The co-planar signal strengths can then be corrected within their respective planes by exploiting the trigonometric identity, $A=A \cos ^{2}(x)+A \sin ^{2}(x)$ :

$s_{b}=\left(a^{2}+b^{2}\right)^{\frac{1}{4}}, \quad s_{e}=\left(c^{2}+d^{2}\right)^{\frac{1}{4}}$

These two signal strengths must then be combined, depending on the angular offsets from the opposite plane, using the photodiode model. The bearing based offset angle $(\psi)$ is used to correct the elevation based signal strength $\left(s_{e}\right)$, and the elevation based offset angle $(\phi)$ is used to correct the bearing based signal strength $\left(s_{b}\right)$. Thus, the sphere-tosphere signal strength $(s)$ can be defined as:

$s=\frac{s_{e}}{2 \cos (\psi)}+\frac{s_{b}}{2 \cos (\phi)}$

This sphere-to-sphere signal strength $(s)$ can then be converted to a sphere-to-sphere range $(r)$ using a linear interpolator and a look-up table.

The bearing $(\Psi)$ and elevation $(\Phi)$ angles of the transmitting sensor are then determined by the offset angles $(\psi$ and $\phi)$, the photodiode geometric spacing $(\beta)$ and the photodiode index $(j)$, all with respect to the corresponding plane:

$\Psi=\psi+\beta \times j, \quad \Phi=\phi+\beta \times j$

where $j$ can range from $0:(N-1)$.

This method explains how the relative range, bearing and elevation to a transmitting robot can be estimated using multiple signals from a known geometric photodiode spacing. 
As the algorithm calculations are relatively simple, the processing can be implemented easily on a micro-controller.

\subsection{Coordination among multiple sensors}

Since the goal is to use this sensor for collective operation, signal interference from multiple infrared transmissions must be prevented, thus only one sensor at a time can be transmitting. For dynamic scalability, communication algorithms such as Carrier Sense Multiple Access (CSMA) can be implemented (Pugh et al. 2009). Such algorithms provide a maximum robot density in a given area, which is dependant on the operational update-rate. This link between robot density and operational update-rate is the key limitation of any sensor designed for collective operation. Therefore, increasing the update-rate of the sensor allows for an improved robot density. As the development of scalable communication algorithms is out of the scope of this paper, the focus here is on developing higher operational update-rates. Thus, a simple turn-taking algorithm is employed, where each sensor takes a turn to transmit while the others listen. This is done by creating time slots, where each time slot is allocated a number that repeats every sensor cycle. Each sensor communicates with the others to coordinate the transmission sequence and allow for synchronisation.

The existing infrared relative positioning sensors (McLurkin and Smith 2004; Pugh et al. 2009; Melhuish and Welsby 2002; Kelly and Keating 1996) encode the communication data directly into the infrared signal. However, as the data encoding takes an extended time, this slows down the sensor cycle and increases the complexity of both the transmitter and receiver electronics. To mitigate these problems the data communication is separated from the infrared signal and a simple radio transceiver is used to send the communication data. This allows for a faster infrared signal sampling, thus reducing the sensor cycle time and effectively increasing the update-rate of the sensor by a factor of four when compared to the fastest existing 2-D sensor (Pugh et al. 2009). The radio transceiver is also used to communicate the inverse bearing angles of the receiving robot, as perceived by the transmitting robot. This allows the 3-D position and yaw orientation to be estimated between any two robots within the swarm.

The number of time slots can be dynamically adapted to accommodate any number of sensors. The only limit here is that the update-rate is reduced by adding more time slots. However, as the update-rate of the sensor is fast $(1 \mathrm{kHz})$, the developed simple turn taking algorithm can provide enough speed $(10 \mathrm{~Hz}$ per robot) for a swarm of up to 100 flying robots.

\subsection{Infrared transmission}

In order to obtain the best range, bearing and elevation measurements, it is necessary to evenly emit infrared light in all directions, the more evenly the light is spread, the less the error will be if the sensor is rotated during operation. A constant current source is required to keep the radiated power at a constant level. This current source needs to be pulsed at a defined carrier frequency, which is activated on demand during a communication time slot. The carrier frequency allows the received signal to be bandpass filtered to reject other frequencies that are emitting on the same wavelength. To reduce the number of driver components, arrays of emitters can be used, where one transistor current source can drive several serially connected emitters.

\subsection{Infrared reception}

An important part of the sensor operation is the infrared reception, as the performance of the sensor depends on the quality of the received signal strength. The infrared signal strength is approximately proportional to the inverse square of the range (Naboulsi et al. 2005). The small signal produced by a photodetector at large distances can be in the nano-amps range. The noise introduced by the amplifier itself at high gains can start to dominate over this small signal. Thus, the signal-to-noise ratio can be diminished. The slope of this signal is directly related to the attainable range resolution, which reduces rapidly as the distance increases. This means that it is difficult to obtain a high dynamic range over a long distance.

In order to obtain the infrared signal strength, most researchers are using an FM audio radio circuit, which has been modified for infrared reception (McLurkin and Smith 2004; Pugh et al. 2009; Melhuish and Welsby 2002; Kelly and Keating 1996). The Radio Frequency (RF) chip used is equipped with a Received Signal Strength Indicator (RSSI) pin, which outputs an analogue voltage corresponding to the strength of the received signal. These chips are designed for audio radios, so they operate in the $\mathrm{MHz}$ band. This is a problem as it requires complex RF circuitry that can cause interference in other nearby electronics. The RSSI is normally used on an FM radio only to indicate when a radio channel is tuned in correctly. When modified and used as an infrared signal strength measure, the signal tends to have a relatively small non-linear dynamic range.

To overcome these problems a new technique is proposed involving cascaded filtering, which improves the dynamic range of the signal by segmenting the range space into smaller complementary regions, where each region is covered by a specific amplifier and filter stage with a relatively low gain (to reduce the noise introduced by the amplification process). By doing this the signal becomes more linear, the signal-to-noise ratio of the sensor is improved by highly selective filtering, and the resolution is expanded (see Fig. 2).

A block diagram of the infrared reception path is shown in Fig. 3. The photodiode converts the infrared light into 
small electrical currents that are then pre-amplified. This small modulated signal is fed into a four stage cascaded amplifier. The gain of each amplifier is precisely tuned to cover a specific range segment. On each stage of the cascade there is a highly selective $( \pm 3 \mathrm{kHz})$ bandpass filter tuned to only pass the carrier frequency $(455 \mathrm{kHz})$ of the transmitter. Half-

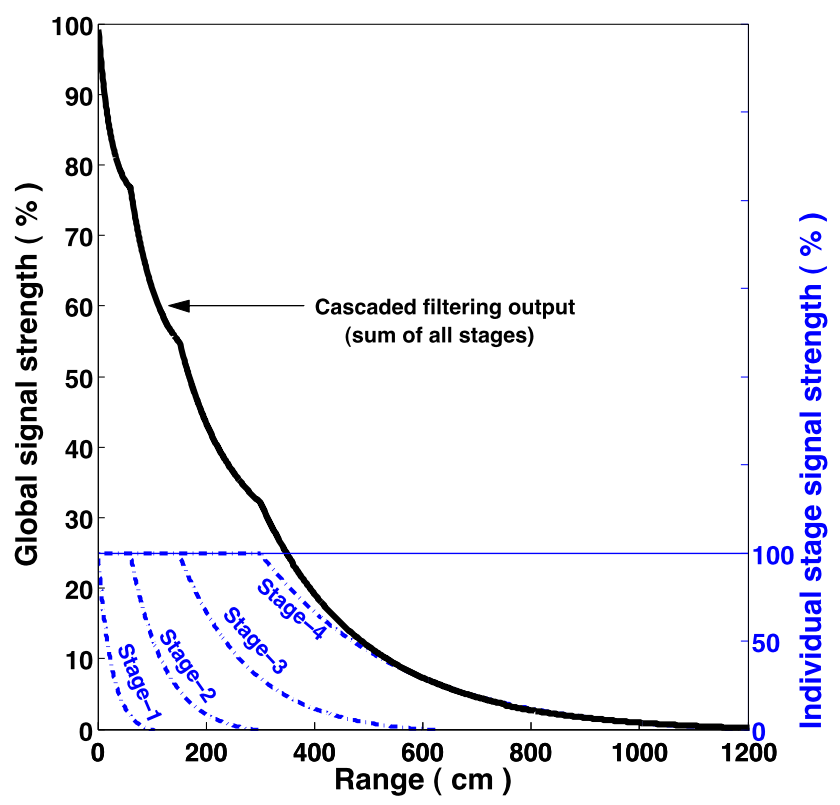

Fig. 2 Theoretical signal strength of the cascaded filtering output (solid line) with respect to the range. The four individual stage outputs of the cascaded filter before they are added together are shown as dashed-dotted lines where each represents $25 \%$ of the total global signal wave rectifiers (Lander 1993) are then used as peak detectors to convert these four signals into DC voltages. These four DC voltages, corresponding to complementary regions of the range space, are then sampled by a 10-bit ADC (provided by the micro-controller) and are added together to obtain the received signal strength. A look-up table is created by collecting the signal strength measurements at different known distances. A linear interpolator is then used to convert the signal strength into a range estimation. This linearisation is essential for this technique, as the summation of all the stages creates small ripples in the global signal strength measurement. Filtering is performed on each stage to improve the signal-to-noise ratio, make the sensor more frequency selective and improve motor interference rejection on the flying robot.

\section{Physical constraints and possible layouts}

The physical implementation of the sensor can be varied depending on the particular application. The size of the sensor can be defined as a function of the operating speed and the individual components required for range sensing. The time delay $(\tau)$ of the cascaded filter response limits the maximum operating speed to $\frac{1}{\tau}$. To achieve this maximum speed, it is necessary to have a cascaded filter for every photodiode. Alternatively, for a reduced size it is also possible to multiplex a single cascaded filter to many photodiodes, in this case the speed $\left(f_{r}\right)$ is determined by dividing the cascaded filter speed by the number of photodiodes that have been multiplexed. Assuming that a cascaded filter having a time

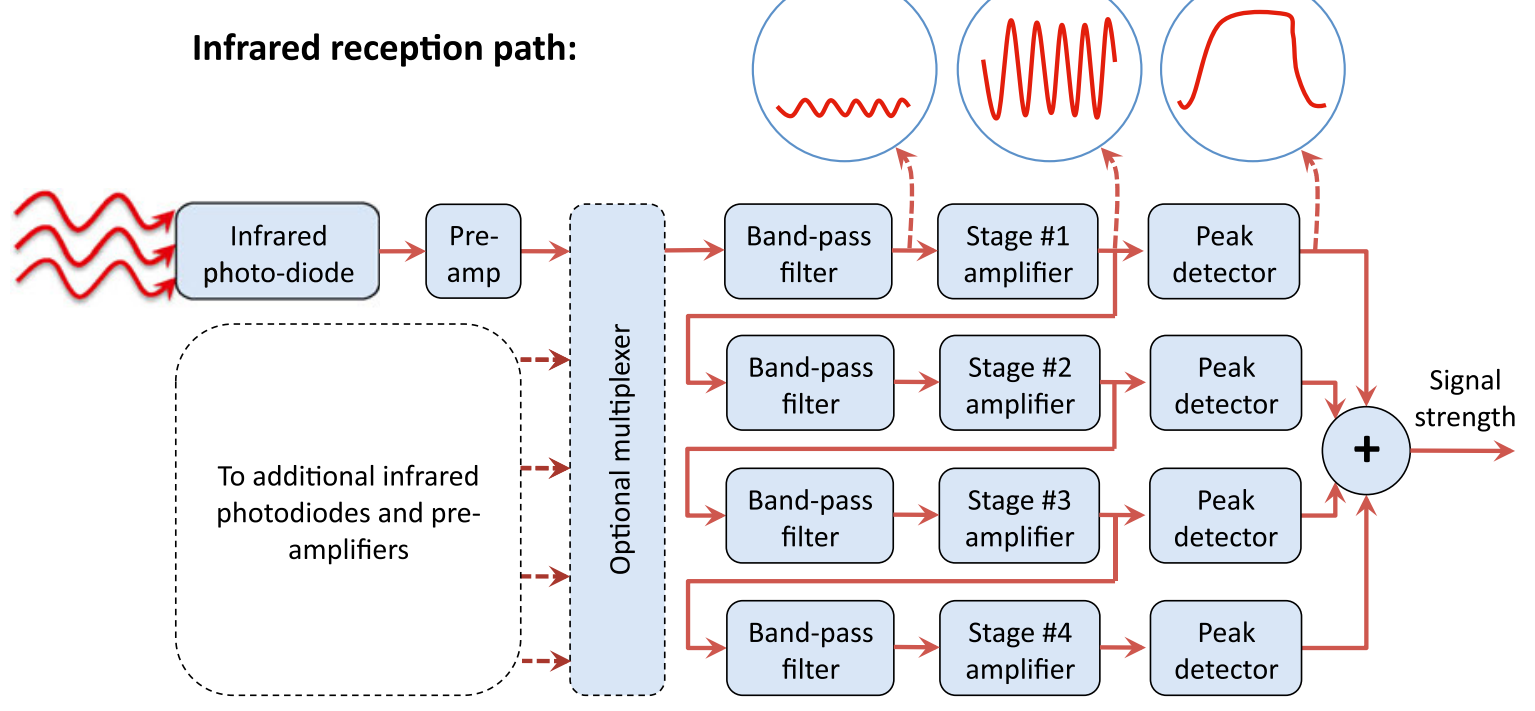

Fig. 3 Reception block diagram showing the infrared reception path of the cascaded filtering method. Stage gains from 1:4 are 22, 15, 13 and 13, respectively. Bandpass filter insertion loss is $-6 \mathrm{~dB}$ 
Table 1 Size and weight of individual parts of the sensor

\begin{tabular}{lll}
\hline Part & Size $\left(\mathrm{cm}^{2}\right)$ & Weight $(\mathrm{g})$ \\
\hline Photodiode and pre-amplifier & $A_{d}=1$ & $m_{d}=0.4$ \\
Cascaded filter and peak detector & $A_{c}=4$ & $m_{c}=4$ \\
Single emitter circuit & $A_{e}=0.5$ & $m_{e}=0.15$ \\
Multiplexer & $A_{m}=2$ & $m_{m}=0.5$ \\
Processor & $A_{p}=4$ & $m_{p}=1$ \\
RF transceiver & $A_{t}=4$ & $m_{t}=1$ \\
\hline
\end{tabular}

delay of approximately $1 \mathrm{~ms}$ is used, the maximum operating speed would be $1 \mathrm{kHz}$. The approximate size and weight of the individual parts needed for operation, can be defined experimentally based on the required surface mount components, shown in Table 1.

Using these values, it is possible to create a model to predict the size $\left(A_{s}\right)$ and weight $\left(m_{s}\right)$ of the sensor based on its operating speed:

$$
\begin{aligned}
A_{s}= & A_{d} \cdot n_{d}+A_{c} \cdot n_{c}+A_{e} \cdot n_{e}+A_{m} \cdot n_{m}+A_{p} \cdot n_{p} \\
& +A_{t} \cdot n_{t}\left(\mathrm{~cm}^{2}\right) \\
m_{s}= & m_{d} \cdot n_{d}+m_{c} \cdot n_{c}+m_{e} \cdot n_{e}+m_{m} \cdot n_{m}+m_{p} \cdot n_{p} \\
& +m_{t} \cdot n_{t}(\mathrm{~g})
\end{aligned}
$$

where $n_{d}$ is the number of photodiodes, $n_{c}$ is the number of cascaded filters, $n_{e}$ is the number of emitters, $n_{m}$ is the number of multiplexers, $n_{p}$ is the number of processors and $n_{t}$ is the number of transceivers used.

Two examples that use this model to show a possible 2-D and 3-D sensor layout are discussed here. For a 2-D sensor, with 8 photodiodes and 16 emitters having a planar $360^{\circ}$ sensor coverage, a single cascaded filter could be used to keep the size small. This would give an operating speed of $f_{r}=\frac{1}{8} \mathrm{kHz}$, or $125 \mathrm{~Hz}$. The size of the sensor would be approximately $30 \mathrm{~cm}^{2}$, or a circle with a $6 \mathrm{~cm}$ diameter, with a weight of $12.1 \mathrm{~g}$. For a 3-D sensor, with 26 photodiodes and 74 transmitters (as shown in Fig. 1) having a full spherical sensor coverage, a cascaded filter for every photodiode could be used to achieve the maximum operating speed $\left(f_{r}=1 \mathrm{kHz}\right)$. The size of the sensor would be approximately $175 \mathrm{~cm}^{2}$, or a circle with a $15 \mathrm{~cm}$ diameter, with a weight of $127.5 \mathrm{~g}$. This physical sensor model can be used as a guide, showing that a 2-D or 3-D sensor can be customised for different physical implementations.

\section{Integration on a flying robot}

In order to achieve unobstructed 3-D sensing on a hovering platform, such as a quad-rotor, the physical construction of the sensor must be considered. The minimum size of a 3-D sensor using the proposed technique has been determined, in
Sect. 3, as a $15 \mathrm{~cm}$ circle with a weight of $127.5 \mathrm{~g}$. However, for all hovering platforms it is important not to disrupt the airflow of the propellers. Disturbing or blocking the airflow will affect the flight characteristics and reduce the efficiency. Thus, this presents a challenging practical implementation problem. One solution is to increase the size of the platform so that there is enough space in the centre for the sensor. However, to achieve unobstructed sensing the sensor would have to be split in two, to cover the top and bottom hemispheres. This implementation is not practical if additional mechanics or sensors need to be added to either the top or bottom of the platform, which is often the case. Additionally, this goes against the philosophy of keeping the platform size as small as possible for indoor operation. One solution that is suitable for unobstructed spherical sensing, is to create a circular sensing ring around the perimeter of the platforms structure. Such a solution, if designed well, could also be used to protect the propellers. By placing the sensor electronics around the perimeter, the sensor geometry is like a compressed sphere, where the sensor angles are kept the same as in the normal spherical geometry. This physical implementation of the 3-D relative positioning sensor has been constructed, as shown in Fig. 4.

The sensor ring must be robust to small collisions and easy to replace, suggesting that a modular design would be suitable. Thus, the sensor was designed in modular sections, where eight sensor sections combine to create one complete ring. For mechanical support two sections are placed in a sandwich configuration, where each sensor section is identical and is designed to plug into a second inverted sensor section, which then covers $1 / 4$ th of the sphere. As the sensors are placed around the circular perimeter of the flying robot, additional photodiodes are required to prevent sensing occlusion caused by the robots structure. On each sensor section there are 4 transmitting and 2 receiving arrays, whose size is defined by the angle of half intensity of the emitters $\left( \pm 25^{\circ}\right)$ and half angular sensitivity of the photodiodes $\left( \pm 60^{\circ}\right)$, respectively. These arrays provide a $1 / 8$ th $\left(90^{\circ}\right.$ wedge) coverage of the sphere. The holders of the transmitter and receiver arrays are made of 3-D printed ABS plastic.

The transmitter arrays each support 5 infrared emitters (Vishay TSAL4400) that are spaced at $22.5^{\circ}$ angles from $0^{\circ}$ along the circular perimeter, to $90^{\circ}$ either upward facing (top sections) or downward facing (bottom sections). Each array can be dynamically controlled allowing for the emission pattern to be altered depending on the user requirements. For the characterisation tests in this paper, all emitters have been activated except for the top $90^{\circ}$ emitters (on the top sections) and bottom $90^{\circ}$ emitters (on the bottom sections) of each transmitter array. This has been done to reduce the environmental reflections from the floor and the ceiling. Additionally, on each top and bottom array pair, there are two redundant emitters facing the same direction, 


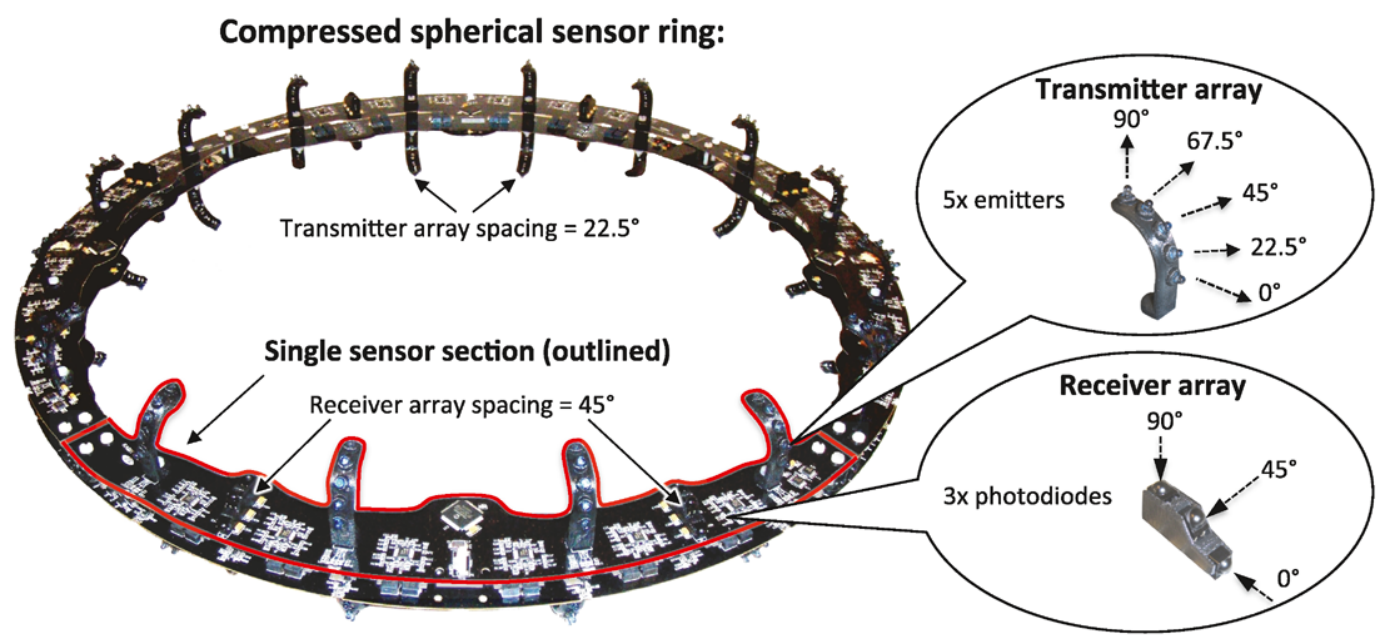

Side view of two sections connected together:

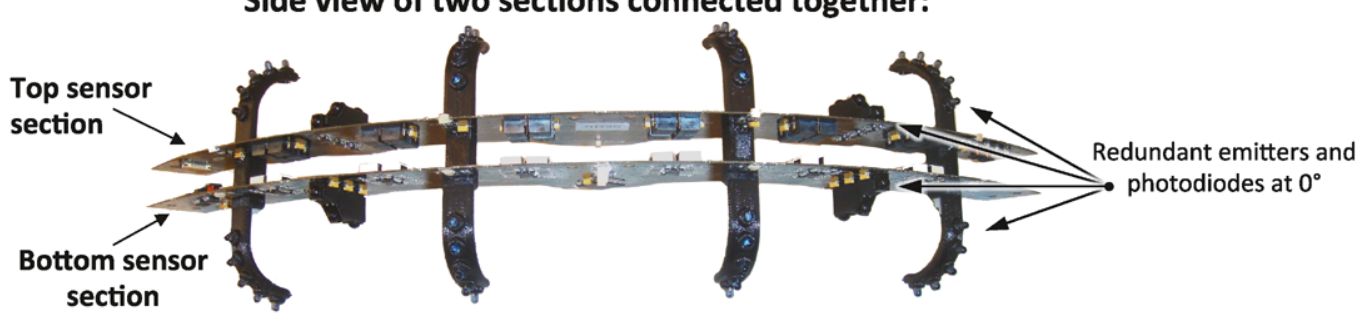

Fig. 4 Top-left: A complete compressed spherical sensor ring, showing eight sensor sections connected together. Top-right: Close-up of a transmitter array and receiver array. Bottom: Side view showing how

pointing $0^{\circ}$ outwards from the perimeter of the sensor. One of these two emitters has also been deactivated to ensure an even emission pattern. The resulting emission pattern of the compressed spherical sensor ring is shown in Fig. 5. The pattern has been estimated by modelling the radiant intensity pattern of the emitter, ${ }^{2}$ and creating a matrix of the summed intensity values over the bearing and elevation angles of the sphere geometry. Only one side of the sphere is shown as the pattern is symmetrical around the perimeter of the sensor. The emission deviation between $\pm 47.5^{\circ}$ elevation, which would relate to quite aggressive flight manoeuvres, is less than 5\%. Beyond these angles, in the direction of the top and bottom of the ring, the emission intensity reduces to around $57 \%$. This means that with this configuration the sensor will not perform well beyond these limits.

The receiver arrays each support three infrared photodiodes (Vishay BPV22NF) spaced at $0^{\circ}$ along the circular perimeter, $45^{\circ}$ and $90^{\circ}$ either upward facing (top sections) or downward facing (bottom sections). The additional photodiodes are highly redundant, however the 'a-like' signals can be averaged to improve the signal-to-noise ratio. They are necessary for this particular arrangement in order to prevent sensing occlusion and allow for a modular sensor

\footnotetext{
${ }^{2}$ http://www.vishay.com (TSAL4400, accessed Feb. 2011).
}

the top and bottom sensor sections connect together, and the location of the redundant photodiodes and emitters

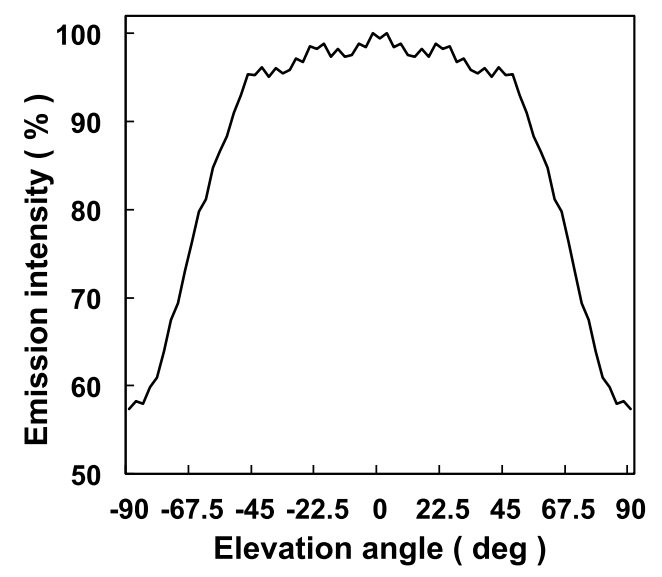

Fig. 5 Emission pattern of one symmetrical side of the compressed spherical sensor ring, where the $0^{\circ}$ elevation is aligned with the outside perimeter of the ring

design. Furthermore, the additional photodiodes at $0^{\circ}$ and $\pm 90^{\circ}$ are beneficial for improved long-range sensing, and floor-to-ceiling altitude regulation (when proximity sensing is used), respectively.

Therefore, the sensor ring has a total of 48 photodiodes (16 receiver arrays) and 160 configurable emitters ( 32 transmitter arrays). To obtain the best signal-to-noise ratio from the sensors, sampling and linearisation of the four cascaded 
outputs is done by a micro-controller placed directly on each section. The measurements are then sent to a main microcontroller for processing.

Using the physical sensor model in Sect. 3, the physical attributes of the sensor electronics can be predicted. The sensor should operate at the maximum speed $(1 \mathrm{kHz})$, therefore each photodiode must have a dedicated cascaded filter. The size of the sensor would be approximately $360 \mathrm{~cm}^{2}$ (with a weight of $245.2 \mathrm{~g}$ ) or a circle with a $22 \mathrm{~cm}$ diameter. This translates into a two layer sensor ring with approximately $48 \mathrm{~cm}$ internal diameter and $50 \mathrm{~cm}$ outer diameter, which would leave only $1 \mathrm{~cm}$ width per side for the electronics. However, this is not practical as the smallest possible width is $2.5 \mathrm{~cm}$, which is defined by the cascaded filter. Also, a ring with these dimensions is too fragile to be mechanically self-supporting. Therefore, the chosen average width is approximately $3.5 \mathrm{~cm}$, which adds an extra $19 \mathrm{~g}$ of mechanical weight for supporting each section. This gives a completed ring weight of approximately $400 \mathrm{~g}$, which now includes rotor protection. The ring can be made larger or smaller depending on the size of the hovering platform. The thin printed circuit boards $(0.6 \mathrm{~mm})$ in the sandwich configuration creates a uni-directional flexible structure that protects the propellers from walls or other large obstacles, but allows for vertical flexing to protect the sensors in the case of a small collision.

The power consumption of the finished sensor while operating at full speed is 10 watts. Comparing this to the typical power required to hover in this size class $(100 \mathrm{~W}$ to $500 \mathrm{~W}$ ), it accounts for only a small percentage ( $2 \%$ to $10 \%$ ) of the total power. However, it is important to note that a considerable amount of power is required to lift the additional weight of the sensor. This additional weight is comparable to a UTM-30LX laser scanner, which is typically used on flying robots (Achtelik et al. 2009; Bachrach et al. 2009; Shen et al. 2011).

\section{Resolution and noise performance}

In order to analyse the sensor's resolution and signal noise, each photodiode of the sensor has been calibrated. For this experiment, one sensor was used as a transmitter, and the other was used as a receiver (see video). ${ }^{3}$ To automate the tests, the receiving sensor was attached to an IRB-140 ABB robotic arm and the transmitting sensor was attached to a wheeled robot (Bonani et al. 2010). A computer was used to remotely control the 6-axis of the $\mathrm{ABB}$ robot and to set the distance of the wheeled robot along a guide rail. The $600 \mathrm{~cm}$ long, suspended, black guide rail was marked every $10 \mathrm{~cm}$ with white lines so that the position could be detected by the

\footnotetext{
${ }^{3}$ Video: http://jfroberts.com/phd (Sensor calibration).
}

wheeled robot's ground sensors. This allowed the transmitting sensor to be automatically displaced from the receiving sensor at $10 \mathrm{~cm}$ increments from $0 \mathrm{~cm}$ to $600 \mathrm{~cm}$. At each position, the ABB robot aligned each of the 48 photodiodes of the receiving sensor with the centre of the transmitting sensor and 25 consecutive samples of the signal strength were recorded at a rate of $10 \mathrm{~Hz}$.

To determine the resolution of the sensor (i.e the difference in ADC values between two distances), the gradient of the signal strength was calculated at each tested distance by taking the difference in signal strength between two adjacent test distances and dividing by the $10 \mathrm{~cm}$ displacement. The mean RMS resolution was $0.4 \mathrm{~cm}$ at distances up to $600 \mathrm{~cm}$ and $0.14 \mathrm{~cm}$ at distances below $200 \mathrm{~cm}$. The worst RMS resolution was $1.1 \mathrm{~cm}$ at distances up to $600 \mathrm{~cm}$ and $0.3 \mathrm{~cm}$ at distances below $200 \mathrm{~cm}$. To calculate the noise in the signal strength, the signal standard deviation was calculated from the 25 samples for each photo-diode at each test distance. The mean RMS signal standard deviation was $3.2 \mathrm{~cm}$ at distances up to $600 \mathrm{~cm}$ and $1.95 \mathrm{~cm}$ at distances below $200 \mathrm{~cm}$. The worst RMS signal standard deviation was $6.66 \mathrm{~cm}$ at distances up to $600 \mathrm{~cm}$ and $2.6 \mathrm{~cm}$ at distances below $200 \mathrm{~cm}$. These measurements were used to calibrate each sensor by creating a look-up table for the range estimation.

\section{Relative positioning error}

In order to analyse the sensor's 3-D relative positioning performance, two relative positioning sensors were used to measure the error in the calculated range, bearing and elevation measurements. For this experiment, one sensor was used as a transmitter, and the other was used as a receiver (see video). ${ }^{4}$ The transmitting sensor was kept at a fixed height and position during the experiment. The receiving sensor was manually placed along a two dimensional vertical grid. The $100 \mathrm{~cm}$ spaced grid consists of six defined distances $(100 \mathrm{~cm}$ to $600 \mathrm{~cm})$, by three defined heights $(0 \mathrm{~cm}$ to $200 \mathrm{~cm}$ ) from the transmitter. The six defined distances were chosen so that the results would span across the full calibration range of the sensors. The three defined heights were chosen based on the assumption of a standard office room ceiling height being $2.5 \mathrm{~m}$. Thus, the usable height between the transmitting and receiving sensors is approximately $2 \mathrm{~m}$. This vertical grid represents the normal operating space for indoor flying robots.

The symmetry in the photodiode spacing every $45^{\circ}$ around the perimeter of the sensor, allows for a reduction in the bearing tests. Therefore, at each of the measurement positions the receiving sensor was rotated and tested at 6

\footnotetext{
${ }^{4}$ Video: http://jfroberts.com/phd (Sensor characterisation).
} 


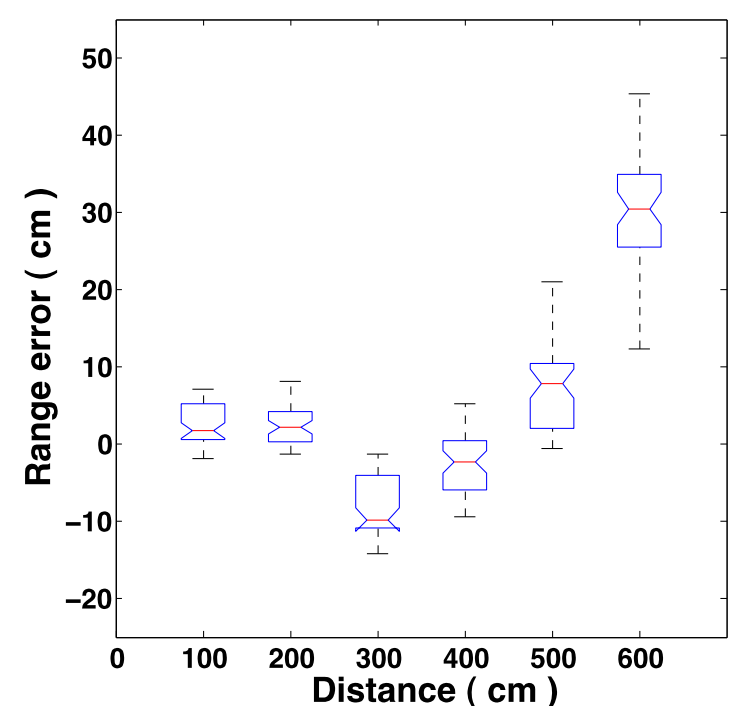

Fig. 6 Box-plot of the range error over the distance, indicating the variance across the samples

different bearing orientations from $0^{\circ}$ to $50^{\circ}$ thus, covering all of the critical bearing angles. For each tested position 50 samples of the received range, bearing and elevation were recorded. During all tests the bearing orientation of the transmitting sensor was continuously rotated backwards and forwards by $90^{\circ}$ using a servo motor. This was to incorporate any transmission errors that are related to non-uniform emissions patterns within the results. During a single measurement of 50 samples, the transmitting sensor would make approximately 5 rotations of $90^{\circ}$. As the calibration was performed automatically with a high-precision robot, and the manual characterisation was aided with laser guides, decimal precision has been used in all experiments.

\subsection{Range performance}

The range response has been measured over the $600 \mathrm{~cm}$ calibration distance of the sensor. The box-plot of the range error over the distance, in Fig. 6, shows the variance across the samples. Analysing the range error shows that the average absolute and relative error was always below 5.02\% $(30.13 \mathrm{~cm}$, measured at $600 \mathrm{~cm})$. The maximum absolute and relative error was always below $7.57 \%(45.40 \mathrm{~cm}$, measured at $600 \mathrm{~cm}$ ).

The influence of the bearing on the range error has been tested at a fixed elevation $\left(0^{\circ}\right)$. The average absolute and relative error was always below $3.06 \%(18.39 \mathrm{~cm}$, measured at $600 \mathrm{~cm}$ ), and the maximum absolute and relative error was always below $7.57 \%(45.40 \mathrm{~cm}$, measured at $600 \mathrm{~cm})$. This indicates that the range error introduced by changes in the bearing is small.

The influence of the elevation (up to $53.1^{\circ}$ ) on the range error has been tested at a fixed bearing $\left(0^{\circ}\right)$. The average absolute error was always below $7.99 \mathrm{~cm}(1.33 \%$, measured at $600 \mathrm{~cm}$ ) and the average relative error was always below $2.71 \%(5.43 \mathrm{~cm}$, measured at $200 \mathrm{~cm})$. The maximum absolute error was always below $35.70 \mathrm{~cm}(7.14 \%$, measured at $500 \mathrm{~cm}$ ) and the maximum relative error was always below $13.01 \%(26.02 \mathrm{~cm}$, measured at $200 \mathrm{~cm}$ ). This indicates that the range error introduced by changes in the elevation is minimal for elevation angles that are $53.1^{\circ}$ or less. However, the range error is larger for elevation angles greater than $53.1^{\circ}$. This is assumed to be due to the compressed sphere geometry of the sensor ring and its non-homogeneous transmission pattern at large elevation angles.

The calibration guide rail length was limited to the size of the experiment room, thus limiting the calibration range to $600 \mathrm{~cm}$. However, the sensor is capable of operating at much further distances. This has been achieved by analysing the trend of the signal strength curve and extending the look-up table manually. The range was measured at a fixed elevation $\left(0^{\circ}\right)$ and a bearing of $22.5^{\circ}$, which is the angle of minimum sensitivity between two infrared photodiodes and represents the worst-case bearing orientation. To determine the reliable operating range of the sensor, the receiver was tested above the calibration range at distance intervals of $200 \mathrm{~cm}$, from $100 \mathrm{~cm}$ to $1100 \mathrm{~cm}$, and again at $1200 \mathrm{~cm}$.

The maximum reliable operating range was found to be at $1200 \mathrm{~cm}$. As the sensor was not calibrated for ranges over $600 \mathrm{~cm}$, the error is not an accurate indication of the maximum performance. However, even without calibration the range error, shown in Fig. 7, is small. Analysing the range error shows that the average absolute and relative error was always below $9.11 \%(109.37 \mathrm{~cm}$, measured at $1200 \mathrm{~cm})$. The maximum absolute and relative error was always below $15.27 \%(183.23 \mathrm{~cm}$, measured at $1200 \mathrm{~cm})$. A summary of the range errors can be seen in Table 3 .

From these results it is clear that there are some systematic range measurement errors as a function of distance. These range skews are likely caused by reflections within the environment, which is more visible over larger ranges due to the smaller respective signal strength. It might be possible to measure the reflections and use them to reduce these errors dynamically. However, it is envisioned that as the error would be more dominant at the source of the transmission, the correction may not be so useful. These reflections would be slightly different in different environments. The only way to guarantee the best performance would be to calibrate the sensor in the actual environment in which it will be used. This could be a simple gain factor adjustment from a measurement taken at $600 \mathrm{~cm}$.

\subsection{Bearing performance}

The box-plot of the bearing error across the 6 tested bearings, shown in Fig. 8, indicates the variance across the samples. Analysing the bearing error shows that the average error was always below $3.13^{\circ}\left(0.87 \%\right.$, measured at $\left.30^{\circ}\right)$, and 


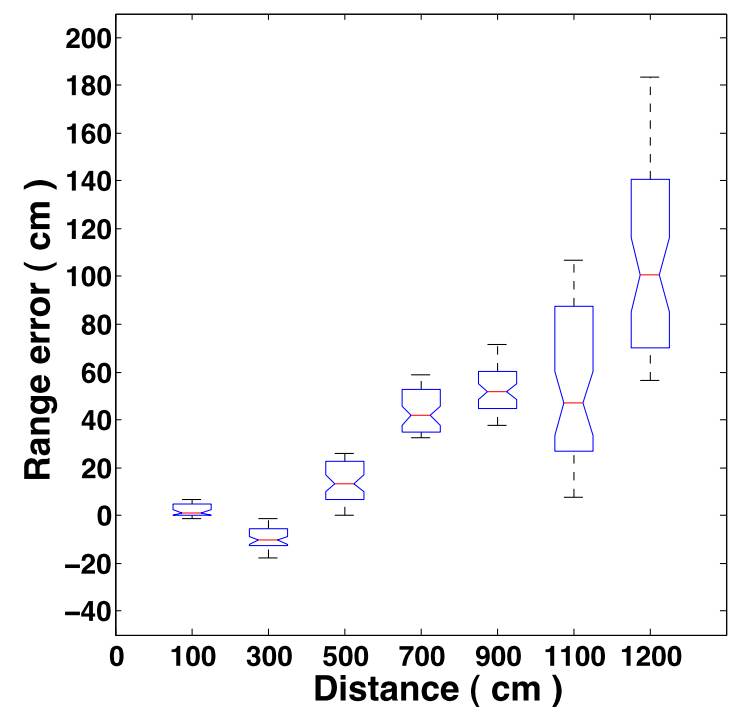

Fig. 7 Box-plot of the range error over the distance, extending beyond the calibration, indicating the variance across the samples at a fixed bearing $\left(22.5^{\circ}\right)$ and elevation $\left(0^{\circ}\right)$

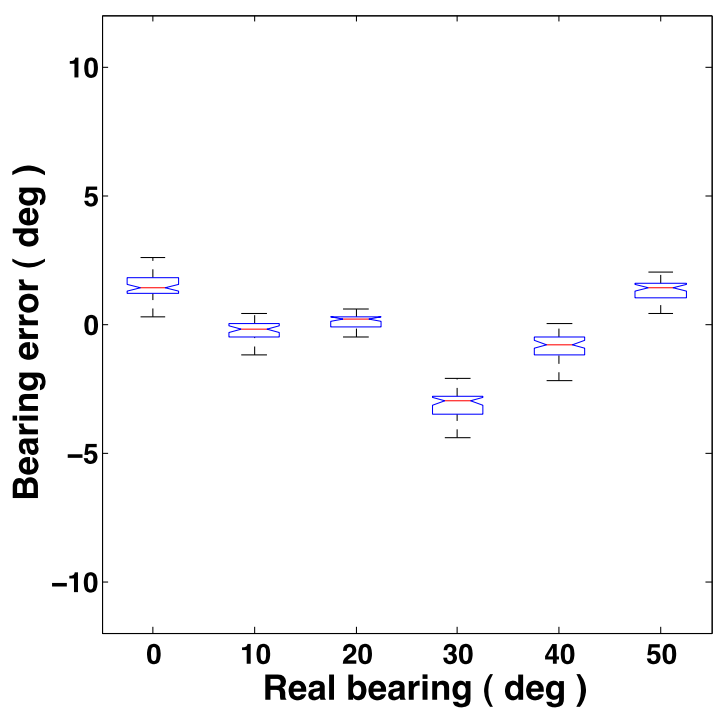

Fig. 8 Box-plot of the bearing error across the 6 tested bearings, indicating the variance across the samples

the maximum error was always below $4.40^{\circ}(1.22 \%$, measured at $30^{\circ}$ ). The observable skew of the bearing at $30^{\circ}$ with a small distribution, relates to the error in the model used to represent the sensitivity response of the photodiode.

The influence of the distance on the bearing error has been tested at a fixed elevation $\left(0^{\circ}\right)$. The average error was always below $3.13^{\circ}$ (measured at $100 \mathrm{~cm}$ and $30^{\circ}$ ), and the maximum error was always below $10.79^{\circ}$ (measured at $100 \mathrm{~cm}$ and $30^{\circ}$ ). This indicates that the bearing error introduced by changes in the distance is small.

The influence of the elevation on the bearing error has been tested over all distances. The average error was al-

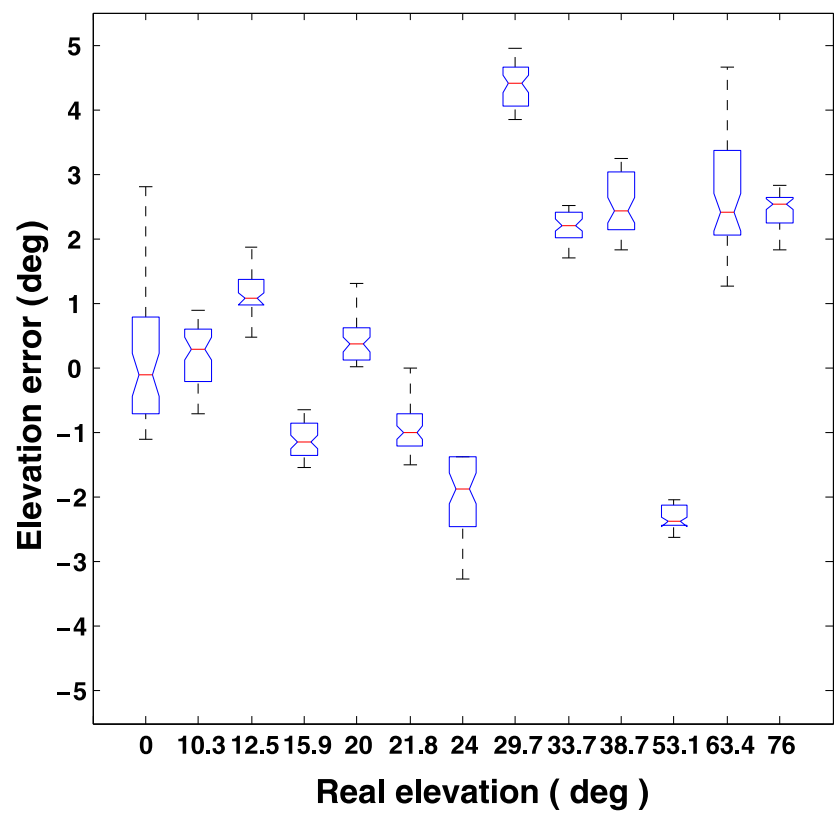

Fig. 9 Box-plot of the elevation error across the 13 tested elevation angles of the sensor, indicating the variance across the samples, which are spread over all distances and heights

Table 2 Elevation angles calculated from the geometry between the sensors

\begin{tabular}{lllllll}
\hline Heights & \multicolumn{5}{l}{ Distances } \\
\cline { 2 - 7 } & $100 \mathrm{~cm}$ & $200 \mathrm{~cm}$ & $300 \mathrm{~cm}$ & $400 \mathrm{~cm}$ & $500 \mathrm{~cm}$ & $600 \mathrm{~cm}$ \\
\hline $200 \mathrm{~cm}$ & $76.0^{\circ}$ & $53.1^{\circ}$ & $38.7^{\circ}$ & $29.7^{\circ}$ & $24.0^{\circ}$ & $20.0^{\circ}$ \\
$100 \mathrm{~cm}$ & $63.4^{\circ}$ & $33.7^{\circ}$ & $21.8^{\circ}$ & $15.9^{\circ}$ & $12.5^{\circ}$ & $10.3^{\circ}$ \\
$0 \mathrm{~cm}$ & $0^{\circ}$ & $0^{\circ}$ & $0^{\circ}$ & $0^{\circ}$ & $0^{\circ}$ & $0^{\circ}$ \\
\hline
\end{tabular}

ways below $2.64^{\circ}\left(0.73 \%\right.$, measured at $76^{\circ}$ elevation $)$, and the maximum error was always below $13.95^{\circ}(3.87 \%$, measured at $33.7^{\circ}$ elevation). This higher value further shows that the model of the photodiodes is not accurate around the $30^{\circ}$ bearing or elevation angles. A summary of the bearing errors can be seen in Table 3 .

\subsection{Elevation performance}

The box-plot of the elevation error across the 13 tested elevation angles (see Table 2), shown in Fig. 9, indicates the variance across the samples spread over all distances and heights. Analysing the elevation error shows that the average error was always below $4.40^{\circ}\left(1.22 \%\right.$, measured at $\left.29.7^{\circ}\right)$, and the maximum error was always below $5.14^{\circ}(1.43 \%$, measured at $33.7^{\circ}$ ). Even though the measurements are scattered due to the varying distances and heights, the error values are similar to the bearing errors.

The influence of the distance on the elevation error has been tested at a fixed bearing $\left(0^{\circ}\right)$. The average error was 
Table 3 Summary of the specifications for the 3-D relative positioning sensor

\begin{tabular}{|c|c|c|c|}
\hline \multicolumn{4}{|c|}{ Operating constraints } \\
\hline Range & $10 \mathrm{~cm}$ to $12 \mathrm{~m}$ & & \\
\hline Bearing & $0^{\circ}$ to $360^{\circ}$ & & \\
\hline Elevation & $0^{\circ}$ to $\pm 76^{\circ}$ & & \\
\hline Speed & $1 \mathrm{kHz}$ & \multicolumn{2}{|l|}{$(\div$ by number of robots $)$} \\
\hline Size & $22 \mathrm{~cm}$ diameter & (single board solution), & $43 / 50 \mathrm{~cm}$ ring diameter (modular solution) \\
\hline Weight & $245.2 \mathrm{~g}$ & (single board solution), & $400 \mathrm{~g}$ (modular solution) \\
\hline \multirow[t]{2}{*}{ Power } & $10 \mathrm{~W}$ & (at full operating speed) & \\
\hline & Average & Worst case & Comment \\
\hline Resolution & $0.40 \mathrm{~cm}(0.14 \mathrm{~cm})$ & $1.1 \mathrm{~cm}(0.3 \mathrm{~cm})$ & $\mathrm{RMS} \leq 6 \mathrm{~m}(\leq 2 \mathrm{~m})$ \\
\hline Noise & $3.20 \mathrm{~cm}(1.95 \mathrm{~cm})$ & $6.6 \mathrm{~cm}(2.6 \mathrm{~cm})$ & $\mathrm{RMS} \leq 6 \mathrm{~m}(\leq 2 \mathrm{~m})$ \\
\hline \multirow[t]{3}{*}{ Range error } & $<5.02 \%$ & $<7.57 \%$ & $\leq 6 \mathrm{~m}$ (across all bearings and $0^{\circ}$ elevation) \\
\hline & $<5.02 \%$ & $<13.01 \%$ & $\leq 6 \mathrm{~m}\left(\right.$ across all bearings and elevations up to $\left.53.1^{\circ}\right)$ \\
\hline & $<9.11 \%$ & $<15.27 \%$ & $\leq 12 \mathrm{~m}$ (fixed bearing and $0^{\circ}$ elevation) \\
\hline \multirow[t]{2}{*}{ Bearing error } & $<3.13^{\circ}$ & $<10.79^{\circ}$ & $\leq 6 \mathrm{~m}$ (across all ranges and $0^{\circ}$ elevation $)$ \\
\hline & $<3.13^{\circ}$ & $<13.95^{\circ}$ & $\leq 6 \mathrm{~m}\left(\right.$ across all ranges and elevations up to $\left.53.1^{\circ}\right)$ \\
\hline Elevation error & $<4.40^{\circ}$ & $<9.40^{\circ}$ & $\leq 6 \mathrm{~m}$ (across all ranges, bearings for elevations to $\left.76^{\circ}\right)$ \\
\hline
\end{tabular}

always below $2.81^{\circ}(0.78 \%$, measured at $600 \mathrm{~cm})$, and the maximum error was always below $9.40^{\circ}(2.61 \%$, measured at $600 \mathrm{~cm}$ ). This indicates that the elevation error introduced by changes in the distance is small.

The influence of the bearing on the elevation error has been tested across all distances. The average error was always below $2.74^{\circ}\left(0.76 \%\right.$, measured at $40^{\circ}$ bearing), and the maximum error was always below $5.74^{\circ}(1.59 \%$, measured at $40^{\circ}$ bearing). This indicates that the elevation error introduced by changes in the bearing is small. A summary of the elevation errors can be seen in Table 3 .

The effect of the elevation angle on the sensor with respect to height and distance displacement, is similar to the effect of attitude tilting during flight translation. Operation indoors requires slow translational speeds therefore, the tilting angle is expected to be low $\left(<10^{\circ}\right)$. However, when the sensors have a height difference, as shown in Table 2 , this elevation angle can quickly increase. Thus, the elevation perceived by the sensor during flight is a function of height, distance and tilt, where the elevation angle with respect to the height and distance geometry is added to the tilting angle during translation.

The elevation error is good $\left(<9.40^{\circ}\right)$ over all the tested elevation angles up to $76^{\circ}$. Therefore, the larger range error for elevation angles above $53.1^{\circ}$ is fully observable, which means that the error could be corrected. Currently, no information about the modified sensor's compressed spherical geometry is used in the algorithm. To further improve the performance of the sensor, the model could be extended to incorporate the modified geometry and assume a sphere sur- face emission, rather than a point emission. Additionally, all errors could be further reduced if the model of the photodiode was improved and a more homogenous emission pattern was achieved. However, the range, bearing and elevation performance is sufficient to provide $3-\mathrm{D}$ positioning and orientation sensing, suitable for enabling goal-directed flight and collective deployment, of highly dynamic flying robots (see video)..$^{5}$

\section{Ambient light robustness}

In order to test the sensor's robustness against large indoor ambient light changes, several measurements were recorded in a room with high-power controllable lighting. The position between the transmitting sensor and the receiving sensor was fixed at $600 \mathrm{~cm}$. Using a light meter, measurements were taken at 0,500, and 10,000 lux, which is equivalent to a dark room, office room, and an overcast day outdoors, respectively. For all of these light levels less than $1 \%$ relative error was observed, therefore the sensor will function correctly over a wide range of realistic indoor environmental lighting conditions.

\section{Proximity sensing}

The sensor is also capable of detecting obstacles using the 3-D proximity sensing. This is achieved by listening to the

\footnotetext{
${ }^{5}$ Video: http://jfroberts.com/phd (Eye-bot tracking).
} 
Table 4 State of the art comparison between top three relative positioning sensors and the developed 3-D sensor. Cells marked in bold indicate the best performing in that category

\begin{tabular}{|c|c|c|c|c|c|c|c|}
\hline \multirow{2}{*}{$\begin{array}{l}\text { Author } \\
\text { reference }\end{array}$} & \multirow[t]{2}{*}{ Ranging method } & \multicolumn{2}{|c|}{ Range error (max) } & \multicolumn{2}{|c|}{ Bearing error $(\max )$} & \multirow{2}{*}{$\begin{array}{l}\text { Update-rate } \\
\text { (10 robots) }\end{array}$} & \multirow{2}{*}{$\begin{array}{l}\text { Operating } \\
\text { range }\end{array}$} \\
\hline & & $3 \mathrm{~m}$ & $6 \mathrm{~m}$ & $3 \mathrm{~m}$ & $6 \mathrm{~m}$ & & \\
\hline $\begin{array}{l}\text { Kemppainen et } \\
\text { al. }(2006)^{\mathrm{a}}\end{array}$ & IR, variable gain & $16.9 \mathrm{~cm}$ & $\mathrm{X}$ & $5.0^{\circ}$ & $\mathrm{X}$ & $0.5 \mathrm{~Hz}$ & $10 \mathrm{~m}$ \\
\hline $\begin{array}{l}\text { Rivard et al. } \\
(2008)^{\mathrm{b}}\end{array}$ & Ultrasound, TOF & $3.0 \mathrm{~cm}$ & $5.0 \mathrm{~cm}$ & $9.0^{\circ}$ & $10.0^{\circ}$ & $1.33 \mathrm{~Hz}$ & $6.7 \mathrm{~m}$ \\
\hline $\begin{array}{l}\text { Pugh et al. } \\
(2009)^{\mathrm{c}}\end{array}$ & IR, RSSI RF chip & $35 \mathrm{~cm}$ & $\mathrm{n} / \mathrm{a}$ & $15.1^{\circ}$ & $\mathrm{n} / \mathrm{a}$ & $25 \mathrm{~Hz}$ & $3.3 \mathrm{~m}$ \\
\hline $\begin{array}{l}\text { Developed 3-D } \\
\text { sensor }^{\mathrm{d}}\end{array}$ & IR, cascaded filtering & $14.2 \mathrm{~cm}$ & $45.4 \mathrm{~cm}$ & $4.3^{\circ}$ & $3.0^{\circ}$ & $100 \mathrm{~Hz}$ & $12 \mathrm{~m}$ \\
\hline
\end{tabular}

${ }^{a}$ Data from Table 2-STD + mean error (transmission spread by mirror)

${ }^{b}$ Data from Fig. 6 (transmission spread by reflector)

${ }^{\mathrm{c}}$ Data from Table 1 and Fig. 8 (including error from rotating transmitter)

${ }^{\mathrm{d}}$ Data from characterisation matching same conditions (including error from rotating transmitter)

reflected signal during a transmission. The angular resolution of the 3-D proximity sensing is defined by the number of photodiodes and their sensitivity angle. The developed 3-D sensor has 48 detectable sectors around the robot in all directions, giving a good proximity coverage. The proximity sensing of one photodiode has been tested on a white, glossy wall and a brown, matte wall. Signal strength measurements were taken at intervals of $10 \mathrm{~cm}$, from 10 to $300 \mathrm{~cm}$. The response, shown in Fig. 10, of the white, glossy wall is stronger than the response of the brown, matte wall, where the maximum detectable range is $300 \mathrm{~cm}$ and $200 \mathrm{~cm}$, respectively. This suggests that the proximity sensing can be used for obstacle detection, but care must be taken in different environments (see video). ${ }^{6}$

\section{Comparison with other relative sensors}

There is a lack of embedded 3-D relative positioning sensors available for a full performance comparison. However, its 2-D features can be compared against the three best performing 2-D relative positioning sensors (with a full $360^{\circ}$ coverage) from the literature (Table 4).

Comparing the developed 3-D sensor against the infrared based 2-D relative positioning sensor by Kemppainen et al. (2006), shows that it is 200 times faster, has a maximum range that is 1.2 times longer, with an accuracy performance that is 1.2 times better for both range and bearing (comparison at $3 \mathrm{~m}$ range due to limited available data). Comparing the developed 3-D sensor against the ultrasonic based 2-D relative positioning sensor by Rivard et al. (2008), shows that it is 75 times faster, has a maximum range that is 1.8

\footnotetext{
${ }^{6}$ http://jfroberts.com/phd (Eye-bot hovering collision).
}

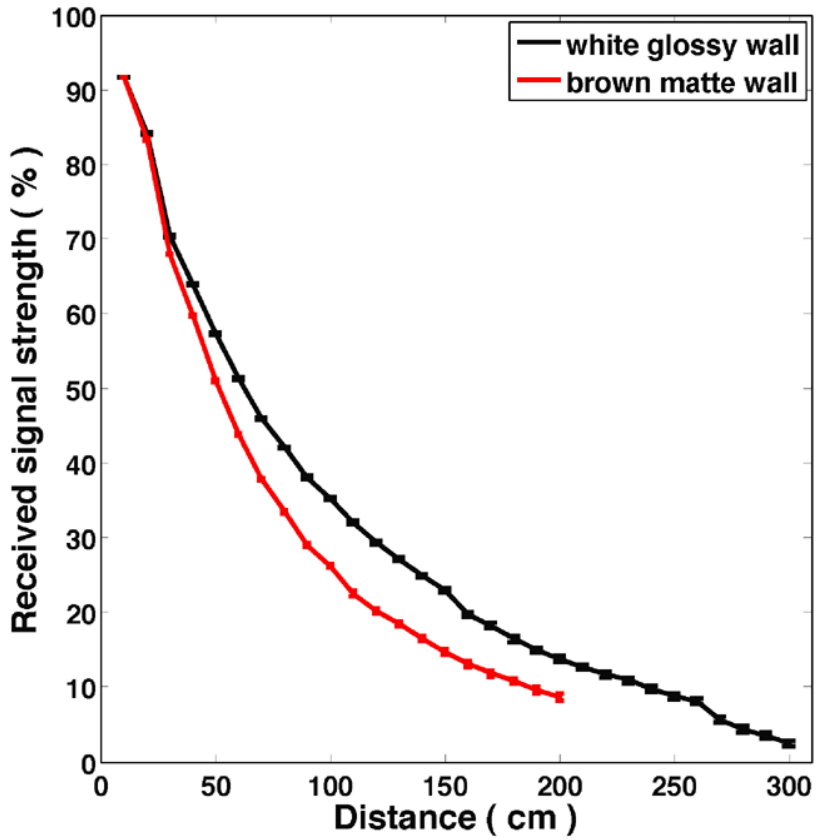

Fig. 10 Proximity sensing showing the range as a function of the reflected signal strength from a single sensing sector, for a white, glossy wall and a brown, matte wall

times longer, with an accuracy performance that is 9 times worse and 3.3 times better for range and bearing respectively (comparison at $6 \mathrm{~m}$ range). Finally, comparing the developed 3-D sensor against the infrared based 2-D relative positioning sensor by Pugh et al. (2009), shows that it is 4 times faster, has a maximum range that is 3.6 times longer, with an accuracy performance that is 2.5 times and 3.5 times better for range and bearing respectively (comparison at $3 \mathrm{~m}$ range). 
The ultrasonic 2-D sensor by Rivard et al. (2008), due to the high accuracy of Time-Of-Flight (TOF) sensing, has the best range error performance. However, the developed 3-D sensor performs better in every other category and has a better range error than the other infrared based sensors. Additionally, the developed sensor is the only sensor capable of providing proximity sensing, 3-D relative positioning and yaw orientation information suitable for indoor flying robots.

\section{Conclusion}

In order to enable the collective operation of indoor flying robots, spatial coordination between individual robots is essential. However, there is a lack of on-board sensing technologies commercially available, or in research that can provide embedded 3-D relative positioning, suitable for the spatial-coordination of indoor flying robots, within realworld environments.

This paper presents a practical on-board sensing method for achieving spatial-coordination between multiple robots in three dimensions. The developed infrared 3-D relative positioning sensor is capable of sensing the range, bearing and elevation between multiple indoor flying robots and can provide proximity sensing in a 3-D space. The developed approach allows for easy adaptation, to suit other robots and applications, depending on a specific sensing speed and coverage requirement. The embedded 3-D relative positioning sensor has the ability to enable inter-robot spatial-coordination in three dimensions, which is necessary for achieving goal-directed flight on highly dynamic flying robots (see video). ${ }^{7}$ This approach does not require computationally expensive algorithms, external sensors or modification of the environment, and is largely independent on varying indoor environmental illumination.

Acknowledgements We would like to thank the people who assisted with the automated calibration system and fabrication of ten 3-D sensor rings: IRIDIA, Université Libre de Bruxelles; Ali Emre Turgut, Arne Brutschy, Manuele Brambilla, Nithin Mathews. LIS, Ecole Polytechnique Fédérale de Lausanne (EPFL); Thomas Schaffter, Peter Dürr, Jürg Germann, Yannick Gasser, Michal Dobrzynski, Yannick Gasser. We would also like to thank Michael Bonani and Philippe Rétornaz for providing valuable feedback during the design phase. Finally, we would like to thank the following people for providing the ABB robot, wheeled robot and mechanical interface: LRSO, EPFL; Lionel Flaction, Tarek Baaboura, Prof. Reymond Clavel, Dr. Francesco Mondada. This work is part of the Swarmanoid project, Future Emerging Technologies (FET IST-022888), funded by the European commission. Additional funding has also come from the Swiss National Science Foundation.

\footnotetext{
${ }^{7}$ Video: http://jfroberts.com/phd (Eye-bot scenario).
}

\section{References}

Achtelik, M., Bachrach, A., He, R., Prentice, S., \& Roy, N. (2009). Stereo vision and laser odometry for autonomous helicopters in GPS-denied indoor environments. In Proceedings of unamanned systems technology XI (SPIE'09), Orlando (Vol. 7332, pp. 19011910). Bellingham: The International Society for Optical Engineering.

Bachrach, A., He, R., \& Roy, N. (2009). Autonomous flight in unstructured and unknown indoor environments. In Proceedings of the 2009 European micro air vehicle conference and flight competition (EMAV'09).

Blösch, M., Weiss, S., Scaramuzza, D., \& Siegwart, R. (2010). Vision based mav navigation in unknown and unstructured environments. In 2010 IEEE international conference on robotics and automation (ICRA) (pp. 21-28).

Bonani, M., Longchamp, V., Magnenat, S., Rétornaz, P., Burnier, D., Roulet, G., Vaussard, F., Bleuler, H., \& Mondada, F. (2010). The MarXbot, a miniature mobile robot opening new perspectives for the collective-robotic research. In International conference on intelligent robots and systems (IROS), 2010 IEEE/RSJ (pp. 41874193). New York: IEEE Press.

Breitenmoser, A., Kneip, L., \& Siegwart, R. (2011). A monocular vision-based system for $6 \mathrm{~d}$ relative robot localization. In Proc. of the IEEE/RSJ international conference on intelligent robots and systems (IROS).

Grzonka, S., Grisetti, G., \& Burgard, W. (2009). Towards a navigation system for autonomous indoor flying. In Proceedings of the international conference on robotics and automation (ICRA'09) (pp. 2878-2883). Piscataway: IEEE Press.

Guenard, N., Hamel, T., \& Mahony, R. (2008). A practical visual servo control for a unmanned aerial vehicle. IEEE Transactions on Robotics and Automation, 24(2), 331-341.

Hoffmann, G. M., \& Tomlin, C. J. (2008). Decentralized cooperative collision avoidance for acceleration constrained vehicles. In Proceedings of the 47th IEEE conference on decision and control, Cancun, Mexico.

Kelly, I. D., \& Keating, D. D. A. (1996). Flocking by the fusion of sonar and active infrared sensors on physical autonomous mobile robots. In Proc. of the third int. conf. on mechatronics and machine vision in practice (pp. 1-4).

Kemppainen, A., Haverinen, J., \& Roning, J. (2006). An infrared location system for relative pose estimation of robots. In Symposium of robot design, dynamics, and control (pp. 379-386).

Kirchner, N., \& Furukawa, T. (2005). Abstract infrared localisation for indoor uavs. In Proceedings of the international conference on sensing technology (pp. 60-65).

Lander, C.-W. (1993). Rectifying circuits. In Power electronics (3rd ed.). New York: McGraw Hill.

Lupashin, S., Schöllig, A., Sherback, M., \& D’Andrea, R. (2010). A simple learning strategy for high-speed quadrocopter multiflips. In Proceedings of the international conference on robotics and automation (ICRA'10) (pp. 642-648). Piscataway: IEEE Press.

McLurkin, J., \& Smith, J. (2004). Distributed algorithms for dispersion in indoor environments using a swarm of autonomous mobile robots. In 7 th international symposium on distributed autonomous robotic systems, Toulouse, France.

Melhuish, C., \& Welsby, J. (2002). Gradient ascent with a group of minimalist real robots: Implementing secondary swarming. In IEEE international conference on systems, man and cybernetics (Vol. 2, pp. 509-514).

Montesano, L., Montano, L., \& Burgard, W. (2004). Relative localization for pairs of robots based on unidentifiable moving features. In Proceedings 2004 IEEE/RSJ international conference on intelligent robots and systems (IROS 2004) (Vol. 2, pp. 1537-1543). 
Naboulsi, M., Sizun, H., \& Fornel, F. (2005). Propagation of optical and infrared waves in the atmosphere. In Proceedings of the union radio scientifique internationale.

Nakamura, T., Oohara, M., Ogasawara, T., \& Ishiguro, H. (2003). Fast self-localization method for mobile robots using multiple omnidirectional vision sensors. Machine Vision and Applications, 14(2), 129-138.

Pilz, U., Popov, A., \& Werner, H. (2009). Robust controller design for formation flight of quad-rotor helicopters. In Proceedings of the 48th IEEE conference on decision and control, held jointly with the 2009 28th Chinese control conference. CDC/CCC 2009 (pp. 8322-8327).

Pugh, J., Raemy, X., Favre, C., Falconi, R., \& Martinoli, A. (2009). A fast on-board relative positioning module for multi-robot systems. In IEEE/ASME transactions on mechatronics, focused section on mechatronics in multi robot systems.

Rivard, F., Bisson, J., Michaud, F., \& Letourneau, D. (2008). Ultrasonic relative positioning for multi-robot systems. In IEEE international conference on robotics and automation, ICRA 2008 (pp. 323-328).

Roberts, J., Zufferey, J.-C., \& Floreano, D. (2008). Energy management for indoor hovering robots. In Proceedings of the international conference on intelligent robots and systems (IROS'08) (pp. 1242-1247). Piscataway: IEEE Press.

Roberts, J., Stirling, T., Zufferey, J.-C., \& Floreano, D. (2009). 2.5D infrared range and bearing system for collective robotics. In Proceedings of the international conference on intelligent robots and systems (IROS'09) (pp. 3659-3664). Piscataway: IEEE Press.

Rudol, P., Wzorek, M., Conte, G., \& Doherty, P. (2008). Micro unmanned aerial vehicle visual servoing for cooperative indoor exploration. In Proceedings of the aerospace conference (pp. 1-10). Piscataway: IEEE Press.

Sahin, E. (2005). Swarm robotics: from sources of inspiration to domains of application. In International workshop on swarm robotics (pp. 10-20).

Shen, S., Michael, N., \& Kumar, V. (2011). Autonomous multi-floor indoor navigation with a computationally constrained mav. In Proc. of the IEEE international conference on robotics and automation (ICRA).

Shoval, S., \& Borenstein, J. (2001). Measuring the relative position and orientation between two mobile robot with binaural sonar. In ANS 9 th international topical meeting on robotics and remote systems, Seattle, Washington.

Soundararaj, S. P., Sujeeth, A. K., \& Saxena, A. (2009). Autonomous indoor helicopter flight using a single onboard camera. In Proceedings of the 2009 IEEE/RSJ international conference on intelligent robots and systems, IROS'09 (pp. 5307-5314). Piscataway: IEEE Press.

Stirling, T., Wischmann, S., \& Floreano, D. (2010). Energy-efficient indoor search by swarms of simulated flying robots without global information. Swarm Intelligence, 4(2), 117-143.

Valenti, M., Bethke, B., How, J.-P., Farias, D.-P., \& Vian, J. (2007). Embedding health management into mission tasking for UAV teams. In American control conference (pp. 5777-5783). Piscataway: IEEE Press.

Weiss, S., Scaramuzza, D., \& Siegwart, R. (2011). Monocular-slambased navigation for autonomous micro helicopters in gps-denied environments. Journal of Field Robotics, 28(6), 854-874.

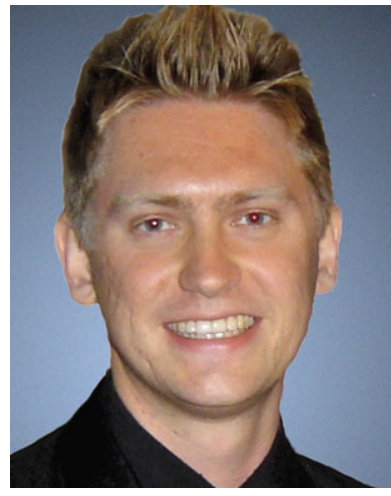

James F. Roberts in June 2004 received a B.Eng. with Honours in Microelectronics Engineering, majoring in Communications Systems from Griffith University, Brisbane. His award winning graduation project with Airservices Australia, entitled "A Design for Air-Traffic Audio Control", now manages $11 \%$ of the world's airspace. In 2007 he received a M.Eng. in Aeronautical, Mechanical \& Mechatronic Engineering at the University of Sydney. In 2011 he completed a Ph.D. at the Laboratory of Intelligent Systems, EPFL Switzerland. His Ph.D. thesis, entitled "Enabling the Collective Operation of Indoor Flying Robots", was part of the European FET Swarmanoid project. He is now the Chief Technology Officer of Intelligenia Dynamics based in Granada, Spain, developing and commercializing intelligent Unmanned Aerial Vehicles.

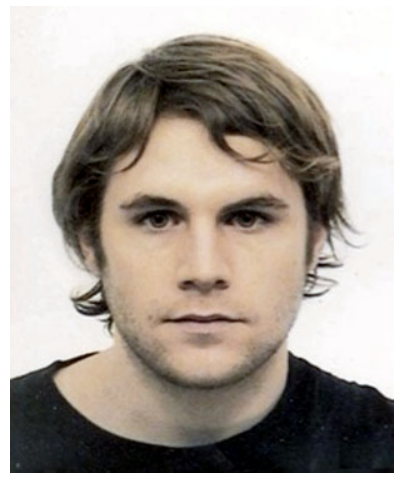

Timothy Stirling in June 2006 received a B.Sc. Joint Honours in Artificial Intelligence and Psychology from the University of Edinburgh, Scotland, where he was awarded the class medal for achievement. From July 2004 to July 2005 he was a Support Engineer providing Product Technical Support for Sun Microsystems Inc., Newark, California. In 2011 he completed a Ph.D. in aerial swarm robotics at the Laboratory of Intelligent Systems, EPFL, Switzerland. He is now a Senior Systems Analyst and Researcher at On Time Systems Inc., Eugene, Oregon, where he applies cutting-edge artificial intelligence algorithms to real-world problems such as real-time traffic routing in dynamic environments.

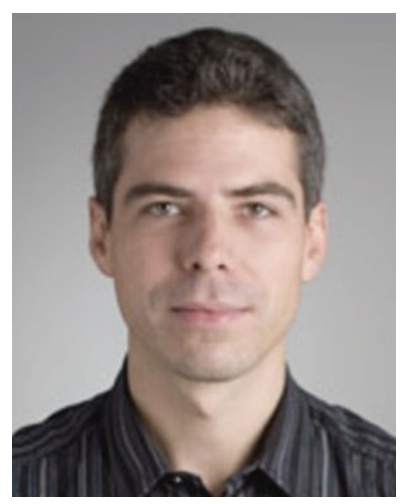

Jean-Christophe Zufferey is group leader at the Laboratory of Intelligent Systems and lecturer in mobile robotics. In 2001 he obtained a Master in micro-engineering from EPFL with a research project carried out at Carnegie Mellon University and in 2005 a Ph.D. in flying robotics from EPFL, for which he received the ABB best Ph.D. award. $\mathrm{He}$ is author or co-author of more than 30 peer-reviewed publications, among which a book on Bioinspired Flying Robots (2008) by the EPFL Press being distributed worldwide by CRC Press. He received the 2006 best paper award at the International Conference on Robotics and Intelligent Systems. In 2007, he coorganized the first international symposium on Flying Insects and Robots with over 100 participants. He is currently coordinating a team of six Ph.D. students working in the design, prototyping, control, and coordination of several small flying platforms equipped with miniature and low-power sensing capabilities. Jean-Christophe is also co-founder of two spin-offs from EPFL. 


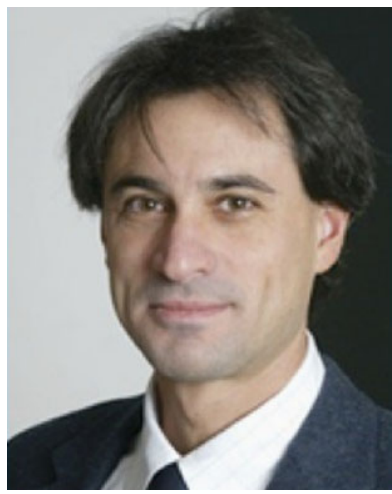

Dario Floreano is Director of the Laboratory of Intelligent Systems within the School of Engineering at Ecole Polytechnique Federale de Lausanne (EPFL). He received an M.A. in Cognitive Science from University of Trieste (Italy) in 1988, an M.S. in Neural Computation from the University of Stirling (UK) in 1991, and a Ph.D. in Evolutionary Robotics in 1995 from University of Trieste (Italy), all of them with distinction. In 1996 he was appointed senior researcher at the Department of Computer Science of EPFL where he established the Robot Learning group. In 1998 he was invited researcher at Sony Computer Science Labs in Tokyo. In 2000 he was awarded a Swiss National Science Foundation professorship at EPFL and in 2005 he was appointed associate professor by EPFL and established the Laboratory of Intelligent Systems. His research interests are Bio-inspired Artificial Intelligence and Robotics. Prof. Floreano is co-founder of the International Society for Artificial Life, Inc., member of the Advisory Group to the European Commission for Future Emerging Technologies, and past-member of the Board of Governors of the International Society for Neural Networks. He published almost 200 peer-reviewed technical papers and edited and co-authored several books, among which Evolutionary Robotics with Stefano Nolfi (hardcover 2000; paperback 2004) and Bioinspired Artificial Intelligence with Claudio Mattiussi (2008), both by MIT Press. He delivered more than 100 invited talks worldwide, co-organized more than 10 international conferences, and is on the editorial board of 10 international journals. 